\title{
State-of-the-art in plant component flow-induced vibration (FIV)
}

\author{
Shuichiro Miwa' (ه), Takashi Hibiki² \\ 1. Graduate School of Engineering, Hokkaido University, Kita 13 Nishi 8, Kita-ku, Sapporo 060-8628, Japan \\ 2. School of Nuclear Engineering, Purdue University, 400 Central Drive, West Lafayette, IN 47907-2017, USA
}

\begin{tabular}{|c|c|}
\hline Abstract & Keywords \\
\hline Flow-induced vibration (FIV) continues to be a critical phenomenon for plant safety. Notably, the & flow-induced vibration (FIV) \\
\hline understanding of FIV generated by multiphase flow is still immature, and various accidents and & fluid-structure interaction \\
\hline troubles have been reported for the plant components including a steam generator, natural gas & two-phase flow \\
\hline lines, piping systems, and so on. It is because FIV is complicated to be predicted during the & slug flow \\
\hline for new types of FIV has been through post-processing by conducting the laboratory-scale & Article History \\
\hline experiment to simulate the prototype. Computational fluid dynamics (CFD) has become a powerful & Received: 31 March 2019 \\
\hline tool to assess FIV, but the approach is still under development for multiphase flow case. It is & Revised: 30 April 2019 \\
\hline partly due to the lack of experimental data, incomplete interfacial transfer terms included in the & Accepted: 30 April 2019 \\
\hline two-fluid model, as well as the difficulty to couple two-phase flow dynamics and structural & \\
\hline dynamics in the simulation stage. Additionally, inadequate FIV database for the simulation & Review Article \\
\hline benchmark also needs to be resolved for the advancement of CFD and finite-element-method (FEM) & (c) The Author(s) 2020, Corrected \\
\hline $\begin{array}{l}\text { models. The present review summarizes fundamentals of FIV caused by gas-liquid two-phase } \\
\text { flow, and recent FIV research activities ranging from experiment to simulation. }\end{array}$ & Publication November 2020 \\
\hline
\end{tabular}

\section{Introduction}

In the plant piping component of energy systems such as heat exchangers and multiphase reactors, noise, vibration, and flow oscillations often become critical problems which may disturb the efficient plant operation. Such fluid-structure interaction problem has led to increased attention in recent years due to the advancement in thermal-hydraulic measurement techniques to capture detailed local phenomena, as well as the much-enhanced computational power to conduct multi-dimensional fluid dynamic phenomena. Ever since the flow-induced vibration (FIV) terminology was introduced by Blevins (1990), much effort has devoted worldwide to solving specific FIV problems. In Japan, research seminar led by the University of Tokyo has been actively carried out since the 1990s to exchange FIV related issues observed in the industry. From the research seminar, the strategic flow diagram to resolve FIV issue was proposed, which is shown in Fig. 1. When the new thermal-hydraulic design is proposed, FIV is usually detected after operating the system for some time. FIV usually shows up as vibration, noise, pressure, and velocity fluctuations. In the worst case scenario, FIV is first noticed as a plant accident which was the case for Monju, the fast breeder reactor in Japan. Hence, in realistic point of view, prediction of FIV is only possible based on the good understanding of the past FIV phenomena. As such, laboratory-scale FIV experiment to develop "FIV database" is quite important to pinpoint exact cause of the FIV. Development of the FIV database is likely the most practical approach to advance "design-aid system for FIV" to conduct R\&D of new plant system. Additionally, due to the advancement of computational power and resource, utilization of CFD and modal analysis tools has become a vital part to simulate FIV phenomena. However, in the case of gas-liquid two-phase flow, constitutive equations utilized in the commercially available codes may not be suitable for the prototypic FIV analysis, and its validity should always be benchmarked by the FIV database. Additionally, state-of-the-art of two-phase flow analysis still has not been well adopted to FIV analysis. For example, the latest FIV handbook published by JSME (2018) does not incorporate recent works including the flow regime transition criteria for large diameter pipe, interfacial area concentration correlations, and so on. This is solely due to the weak linkage between fluid mechanics and structural

The original version of this article was revised due to a retrospective Open Access order.

$\triangle$ smiwa@eng.hokudai.ac.jp 


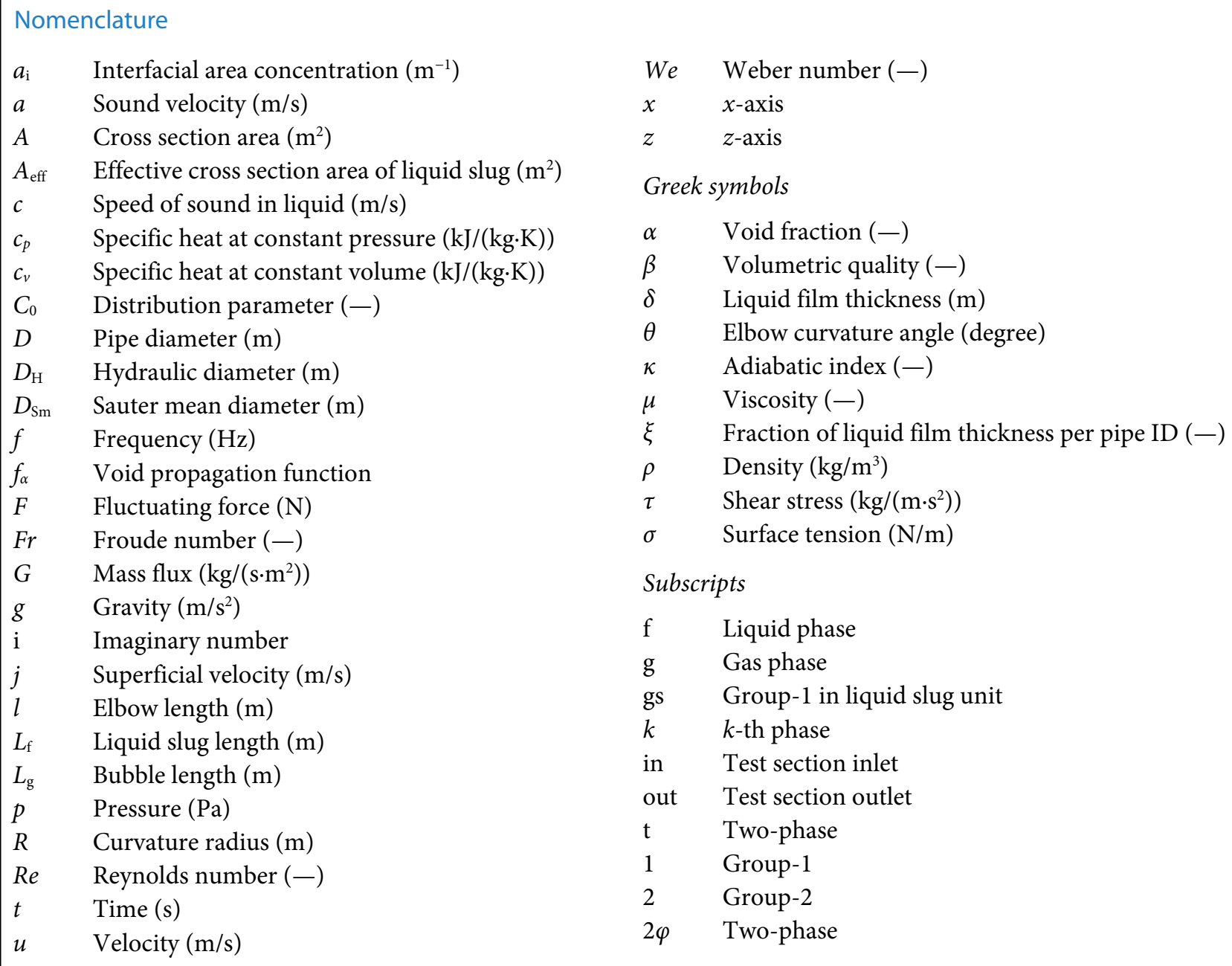

engineering. FIV is a fluid-structure interaction problem, and state-of-the-art of fluid mechanics (including multiphase flow) and structural dynamics should be thoroughly investigated for practical solutions.

According to the recent technical roadmap of FIV phenomena published by Japan Society of Mechanical

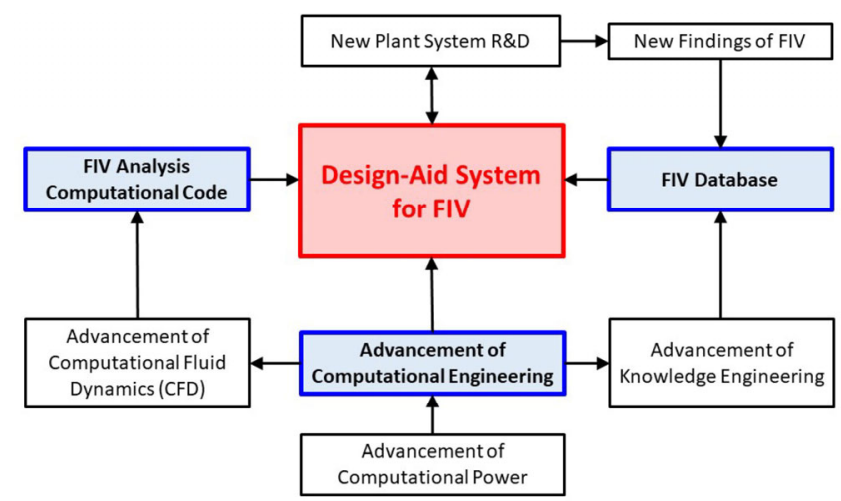

Fig. 1 Design-aid flowchart for industrial FIV phenomena (JSME, 2018; reproduced with permission).
Engineers, problems due to FIV area are becoming significant in the areas of energy industries such as piping systems in nuclear and coal power plants, flow instability in hydroelectric power plant, wind resistivity of solar panels, combustion vibration in biomass plant, noise arose by abnormal ignition in combustion systems, and many more (JSME, 2018; Miwa et al., 2018). Additionally, fluid-structure interaction problems are commonly seen in gas, liquified natural gas (LNG), and petrochemical plants. Solutions to these FIV problems are crucial for safe and efficient operation of energy systems. Key future technologies to resolve FIV problems are listed as follows:

(1) advancement and high efficiency in coupling analysis of fluid-structure interaction problems;

(2) development of predictive and preventative methods of combustion vibration;

(3) active and passive flow controls;

(4) preventative methods for vibro-acoustic problems;

(5) preventative methods of self-excitation due to the vortex generation and swirling flow; 
(6) FIV preventative methods for large-scale facility and high-speed flow applications.

In the current article, recent advancement in internal FIV research in view of industrial applications will be reviewed. Journal publication and international conference presentations following the review article published in 2015 (Miwa et al., 2015) to the present date are mainly covered in this article.

\section{FIV in large-scale plant}

Various types of FIVs exist in large-scale plant. As can be seen from Fig. 2, multiphase flow induced FIV is triggered by various fluid dynamic mechanisms, as well as the flow orientations. In this section, recent studies on FIVs due to multiphase flow in industrial plants and shell-and-tube heat exchangers are reviewed.

In the work carried out by da Silva et al. (2016), FIV in waste heat-recovery steam generator (WHRSG) of the residue fluid catalytic cracking (RFCC) unit was considered by adopting large eddy simulation (LES) using CFD tool. The WHRSG has U-shaped flow channel composed of downward inlet, horizontal and upward outlet channels. Within the upward flow channel, superheaters, evaporator tube bundles, and economizer are installed. FIVs were observed when flue-gas mass flow reached its rated capacity. Four main FIV mechanisms for this facility were pointed out by the authors: fluid elastic instability, periodic vortex shedding, turbulent buffeting, and acoustic resonance. From the LES simulation, generation of the large recirculation zone was confirmed at the joint of horizontal and upward flow channels. This work presented an example of how 3D-CFD simulation tool can be utilized to pinpoint the source of velocity fluctuations without going through mock-up experiment.

FIV analysis using CFD for multiphase separation plants for oil and gas production was introduced by Al-Khalifa et al. (2016). In such type of plant, slug flow can be generated which could damage the piping structure. Interruption of the operation can cause significant economic loss for the oil and gas production company and thorough analysis is vital to prevent such events. As is depicted in their proceeding, multiphase mixture is discharged to the separator through the

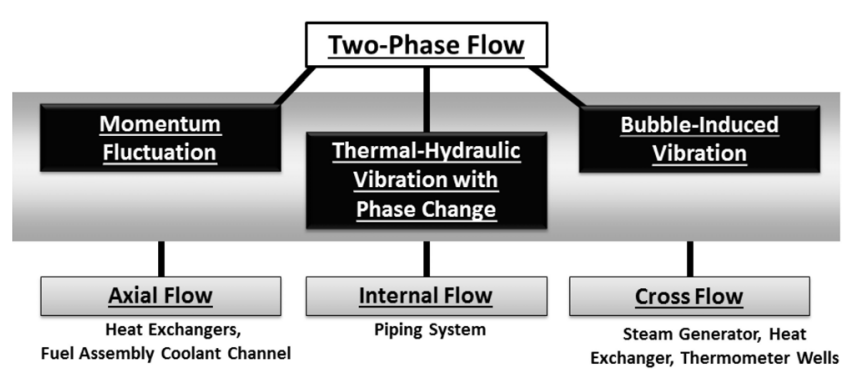

Fig. 2 Mechanisms of two-phase flow induced FIV (Miwa et al., 2015; reproduced with permission (c) Elsevier Ltd. 2014). inlet device in a flow regime of slug, stratified-wavy, or annular flow. In their study, 3D-CFD and 1D transient multiphase simulation package (OLGA) were utilized for the FIV analysis. Impact force model on pipe turning element applied on their CFD simulation for the separator vessel. For the transient analysis performed by OLGA, $1 \mathrm{~km}$ long pipeline with 36 " pipe diameter was considered for the upstream of the separation unit. In the case of sudden startup operation, large spike in liquid hold-up as well as mixture velocity was observed, and these results served as an inlet condition of their 3D-CFD simulation. Three types of inlet designs were considered which include attachment of circular separation unit, vane deflector, and impingement plate. Volume-of-fluid (VOF) and realizable $k$-epsilon models were selected for the analysis. Surface tension effect was ignored, which is a reasonable assumption for the flow regimes beyond slug flow regime (Miwa et al., 2015). Their analysis results showed that the inlet device design plays significant role on the impact force generated by the multiphase flow towards the separator unit. Circulatory phase separator design, which involves abrupt change in flow direction, generated the highest force magnitude onto the whole unit. Discrepancy in the peak force values calculated by the CFD and the industrial standard $\left(\rho A V^{2}\right)$ was observed. While the industrial standard approach provided conservative estimate (overestimates the impact force value), it cannot capture the abrupt change in the force magnitude generated by slug flow regime. Hence, coupling of $1 \mathrm{D}$ and $3 \mathrm{D}$ simulations for two-phase FIV can be a promising approach to conduct more accurate and realistic analysis for the plant component.

Shell-and-tube type heat exchangers are commonly utilized in industrial processes and power plants as heat exchangers and steam generators (SG). In order to achieve high performance, high-velocity fluids are injected into these devices while minimizing the impacts of structural obstacles within flow paths. High-speed flow linked with low natural frequency structure is considered as major sources of FIV. In fact, SGs utilized in pressurized water reactor (PWR) are highly vulnerable to FIVs and problems such as tube wear and rupture are often reported by the nuclear industry (Giraudeau et al., 2013; Olala et al., 2014; Pettigrew et al., 2014; Diwakar et al., 2017).

In recent years, Units 2 and 2 of San Onofre Nuclear Generating Station (SONGS) were permanently shut down after FIV induced leakage from SG tube bundles (Blevins, 2018). Blevins (2018) conducted analysis to determine steam cross flow velocity between $U$ tubes, tube mass per unit length, 
and tube damping factor utilized in the plant. For the assessment of the fluid elastic instability, ASME fluid elastic instability criterion was utilized to obtain critical dimensionless mass damping and reduced velocity (ASME, 1998).

$$
\left.\frac{V_{\text {gap }}}{f D}\right|_{\text {critical }}=C \sqrt{\frac{m(2 \pi \zeta)}{\rho D^{2}}}
$$

Here, $V_{\text {gap }}, f, D, C, m, \zeta, \rho$ are the gap velocity between tube, tube natural frequency, tube outer diameter, dimensionless coefficient, tube mass per unit length, tube dimensionless damping factor, and density, respectively. According to the analysis, SG tubes in SONGS were calculated to be unstable for fluid elastic instability, which subsequently causes FIV.

Álvarez-Briceño et al. (2018) performed FIV experiment for two-phase cross flow in a normal triangular tube bundle. From the experimental results, it was suggested that vibration amplitude is highly dependent on flow regime, which is a function of superficial velocity, physical properties of fluids and phase distribution, for triangular tube bundle. RMS of vibration amplitude was found to be in linear relationship with respect to void fraction up to $75 \%$, which corresponds to near the churn-annular transition range. It was also reported that the vibration characteristics may be explained by normalized force spectra and equivalent force spectrum.

\section{FIV due to internal flow}

For internal FIV, pipe turning elements such as elbow and tee continue to be critical problems in piping system involving multiphase flow for various industrial applications. Piping structure of multiphase flow is usually designed at low natural frequency to cope with thermal-expansion.

Internal two-phase FIV is a crucial problem in subsea pipelines. When the slug flow regime appears in the flowline, it may generate considerable excitation force when collided against the pipe turning element such as elbows, tees, and junctions. The article published by Li et al. (2016) focuses the computational work on gas-liquid two-phase flow through internal pipe component using commercial CFD code coupled with computational structural dynamics (CSD) code, namely ANSYS-CFX and ANSYS-Mechanical. Horizontal pipe diameter of $33.3 \mathrm{~mm}$ with $6 \mathrm{~m}$ long, fixed at two ends was considered. Inlet water and air flow rates were adjusted to the area ratios of 70:30, 60:40, 50:50, 40:60, and 30:70, accordingly. At each simulation, displacement in $X$ and $Y$ directions, Von Mises stress in mid-span point and fixed-end point were numerically determined. The presented simulation model was able to calculate vibration amplitude with respect to internal void fraction value.

Cabrera-Miranda and Paik (2019) carried out a study on the nonlinear planar vibrations of a steel lazy wave riser excited by the slug flow in subsea system (Fig. 3). EulerBernoulli beam model was utilized and the steady plug-flow model was introduced as the rectangular pulse train. Influences of the 2-FIV on the riser's fluctuating characteristics as well as riser's ultimate limit state and major damage for the fatigue limit state were investigated in their numerical study. However, no experimental data were presented to support their calculation results.

Wang et al. (2018a, 2018b, 2018c) conducted resonance analysis for the pipeline-riser system. Purpose of the work was to evaluate the resonance effect caused by developing two-phase flow in the riser. Their pipeline-riser system consists of the experimental facility with $12 \mathrm{~m}$ long downward inclined pipeline attached to the $3.5 \mathrm{~m}$ long vertical turning element and the fluid flows out through the vertical oriented pipeline (riser). Two ends of the riser system were hinged. Right underneath the riser, pressure and displacement sensors were installed to measure the pressure fluctuation as well as the linear displacement. During the two-phase flow experiment, flow regime observation was conducted via visualization. Stratified flow, plug flow, slug flow, and transitional flow conditions were observed in the pipeline. Bubbly and/or churn flows were observed in the riser section. Intense pressure fluctuations were observed for stratified and slug flows, and mainly concentrated in low frequency region ranging from 0 to $1 \mathrm{~Hz}$. Under FEM analysis, a dynamic model with capability to predict axial and bending vibrations was proposed. One of the important two-phase flow parameters, void fraction, was calculated using existing empirical correlations. It was concluded that the elastic coefficient of the supporting foundation has a high influence towards the vibration characteristics of the pipeline-riser system. Additionally, it was suggested that the stratified flow and slug flow regimes should be regarded as the critical flow regimes that may cause the fatigue/failure of the piping system.

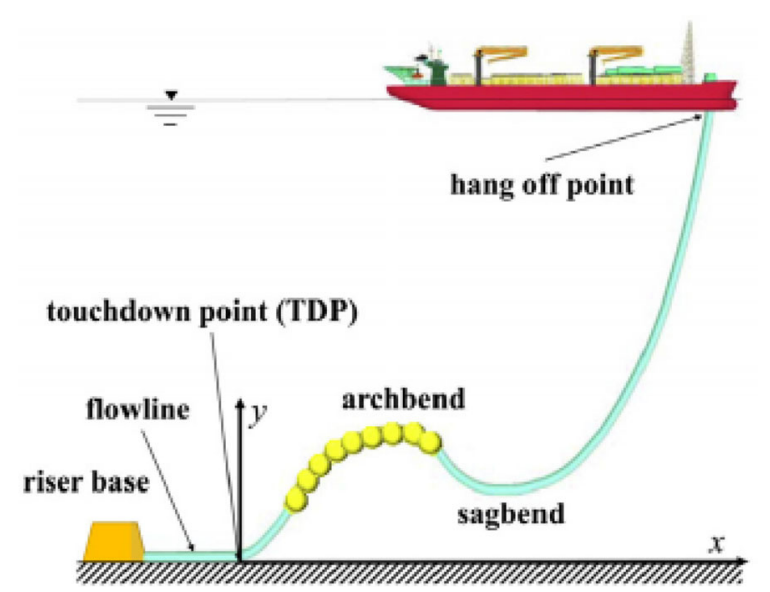

Fig. 3 Schematic of the subsea jumper (Cabrera-Miranda and Paik, 2019; reproduced with permission (C) Elsevier Ltd. 2019). 
Ong et al. (2017) presented a case study on the FIV in oil and gas processing plant equipped with $30 \mathrm{~m}$ long large diameter pipe (24 in). Non-destructive testing approach which includes finite element modal analysis and CFD analysis was utilized to assess the structural integrity. Based on the analysis results, proposal of additional support installation methods along the pipe channel was made.

Nakamura et al. (2005) carried out visualization test on the two-phase flow through large diameter pipe with elbow section. The purpose of the experiment was to simulate the hot-leg and cold-leg pipes of JNC sodium-cooled fast reactor (JSFR) with $1 / 3$ scale. Pressure drop along the elbow section was measured to estimate the turbulence intensity. Maximum FIV was observed at the downstream of the flow separation zone.

Belfroid et al. (2016) performed two-phase FIV experiment with large diameter pipe (6"). Aim of their work was to evaluate the scaling rules from small to large diameter pipe, and to see the effect of upstream disturbance by placing U-bend section at the upstream of the test section. Two types of test sections were utilized in horizontal-upward flow orientation: single elbow and single elbow with U-bend at up-stream (Fig. 4). Pressure transducers, force sensors, and accelerometer were utilized to measure the hydrodynamic force and structural vibration. Similar to the experimental approach taken by Liu et al. (2012), natural frequency of the test section was set much higher than characteristic frequency of two-phase flow to avoid resonance effect. Electrical resistance tomography was utilized to measure area-averaged void fraction. This approach will provide two-dimensional void fraction distribution. Experimental database was developed for the flow regimes ranging from stratified-way to annular two-phase flow.

It was reported that for void fraction spectrum at the upstream of elbow section, correlation proposed by Schulkes (2011) well represented the obtained experimental data. In the correlation, Strohal number $(S t)$ is defined as

$$
S t=\frac{f D}{v_{\mathrm{m}}}=\Phi \cdot \Psi \cdot \Theta
$$

Here, liquid Reynolds number $\left(R e_{\mathrm{f}}\right)$ was utilized to express $\Phi$, which is defined as

$$
R e_{\mathrm{f}}=\frac{\rho_{\mathrm{f}} j_{\mathrm{f}} D}{\mu_{\mathrm{f}}}
$$

Then,

$$
\Phi=12.1 R e_{\mathrm{f}}^{-0.37}
$$

for $\operatorname{Re}_{\mathrm{f}}<4000$ and

$$
\Phi=1
$$

for $R e_{\mathrm{f}}>4000$, respectively. Likewise,

$$
\Psi=0.016 \lambda(2+3 \lambda)
$$

where $\lambda$ is defined as the ratio between superficial liquid velocity $\left(j_{\mathrm{f}}\right)$ and total superficial velocity $(j) . \Theta$ is defined based on Froude number $(F r)$ and pipe inclination angle in $\operatorname{radian}(\theta)$, as follows:

$$
\Theta=1+\frac{2}{F r} \operatorname{sgn}(\theta) \sqrt{|\theta|}
$$

which is valid for the angle of inclination less than 0.17 . Otherwise,

$$
\Theta=\frac{1.8}{F r}\left(0.6+2 \theta-2 \theta^{2}\right)
$$

is recommended. $F r$ is defined as a following expression:

$$
F r=\frac{j_{\mathrm{f}}}{\sqrt{g D \cos \theta}}
$$

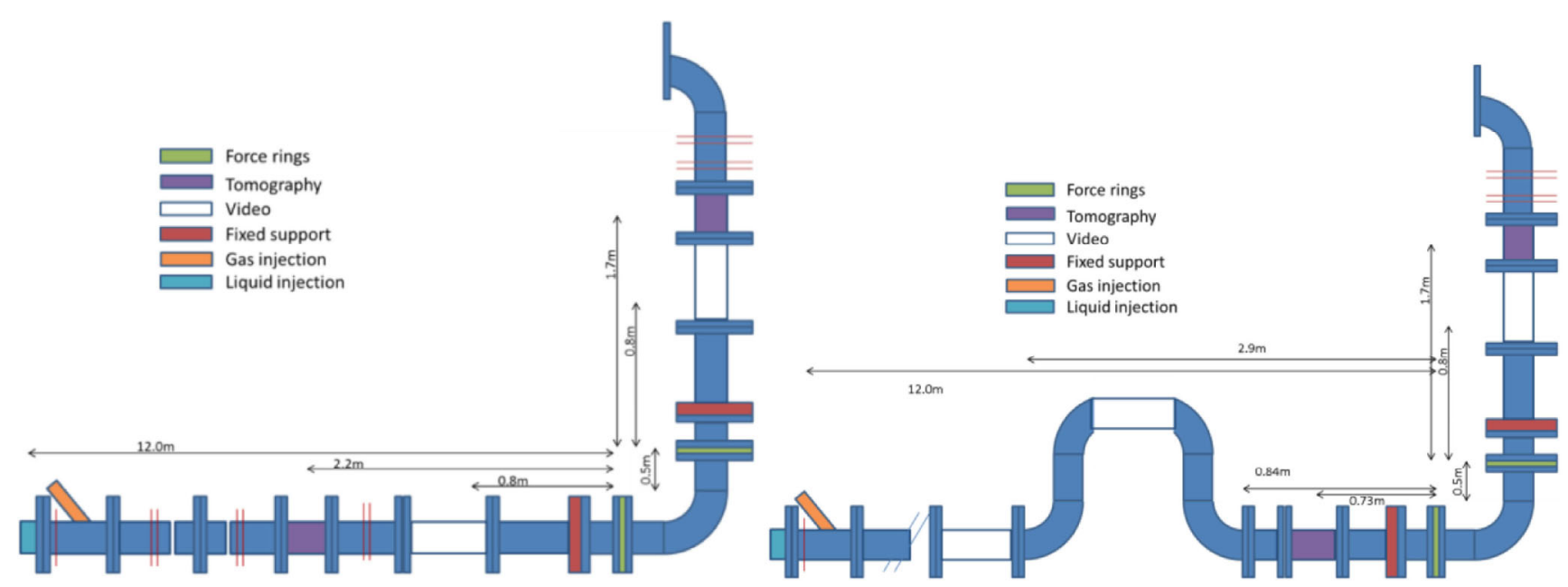

Fig. 4 Investigation of the inlet effect on FIV for the pipe turning element (Belfroid et al., 2016; reproduced with permission ( 
For non-slug flow regime, utilization of LockhartMartinelli parameter well-represented void frequency-based St (Setyawan et al., 2016):

$$
S t=0.25 X^{-1.2}
$$

Here, $X$ is the Lockhart-Martinelli parameter defined as

$$
X=\frac{j_{\mathrm{f}}}{j_{\mathrm{g}}} \sqrt{\frac{\rho_{\mathrm{f}}}{\rho_{\mathrm{g}}}}
$$

In the experiment performed by Belfroid et al. (2016), characteristic frequency of two-phase flow ranges from 0.1 to $5.0 \mathrm{~Hz}$. St tends to approach constant value when $j_{\mathrm{f}}$ increases. Inlet geometry highly affected the gas accumulation behavior at elbow section, and depending on $j_{f}$, elbow either acts as gas-liquid separator or mixer. Root-mean-square (rms) value was utilized to analyze the force fluctuations. The correlation:

$$
F_{\mathrm{rms}}=C\left(\rho_{\mathrm{f}} j^{2} A\right) W e^{-0.4}
$$

with $C=25$ well represented the data for 6 " diameter pipe. The value $C$ changes depending on the pipe diameter size, according to the authors.

For the internal FIV model to calculate structure displacement due to fluid flow, foundation was laid by Paidoussis (1970). One of the advantageous features of having analytical solution for FIV model is that one can assess the stability of the piping system in real and imaginary plane at a given initial velocity. Since then, relevant works have been reported by various researchers. In this section, some of the most recent works are reviewed here.

Wang et al. (2018a) investigated the dynamic behavior of horizontal pipe. In this work, a dynamic model was capable of describing the two-phase slug flow characteristics on a horizontal pipe flow. In order to model the fluid-structure interaction for horizontal slug flow, centrifugal and Coriolis forces were considered, and equation of motion of the pipe structure was solved using finite element method. It was identified that the slug body affects the centrifugal and Coriolis forces, and the structural vibration response is highly linked to the slug unit movement. The maximum loading on the piping structure due to horizontal slug flow is governed by the liquid slug length.

$\mathrm{Xu}$ et al. (2016) derived a non-linear mathematical model to calculate the vibration amplitude of a straight clampedclamped graphite tube for multiphase flow. By utilizing the model, stable/unstable inlet conditions can be identified. Under certain natural frequency conditions, proposed model was capable of predicting vibration amplitude comparable to the experimental data. Critical frequency for the vaporliquid-solid flow was found to be $8.0 \mathrm{~Hz}$ at the flow velocity of $0.78 \mathrm{~m} / \mathrm{s}$.
Ortiz-Vidal et al. (2017) proposed analytical formulation relating hydrodynamic force and structural response. Reasonable results were obtained for the horizontal pipe clamped at both ends of the flow channel. The vibration response was tested for various flow regimes including bubbly to slug flow regimes. Importance of the two-phase damping and hydrodynamic mass was pointed out by the authors. Peak frequency of the vibration response was strongly correlated with hydrodynamic mass value and its value can be reasonably assumed under single-phase fluid theory. For the future works, authors suggested conducting parametric study to assess the effect of boundary and piping length.

\section{FIV for vertical/horizontal slug flow}

From the internal FIV experiment carried out at Purdue University (Fig. 5), force fluctuation model was derived from two-fluid model by Liu et al. (2012). The model was further advanced by Miwa et al. (2014a, 2014b, 2016) by adopting impact force term into the model as well as collecting horizontal two-phase flow database (Fig. 6). In these research work, pipe turning element was considered as one-dimensional beam model and two-phase flow was considered as a mixture. Then, force acting on elbow can be explained by the momentum fluctuation and pressure fluctuation. Gravity

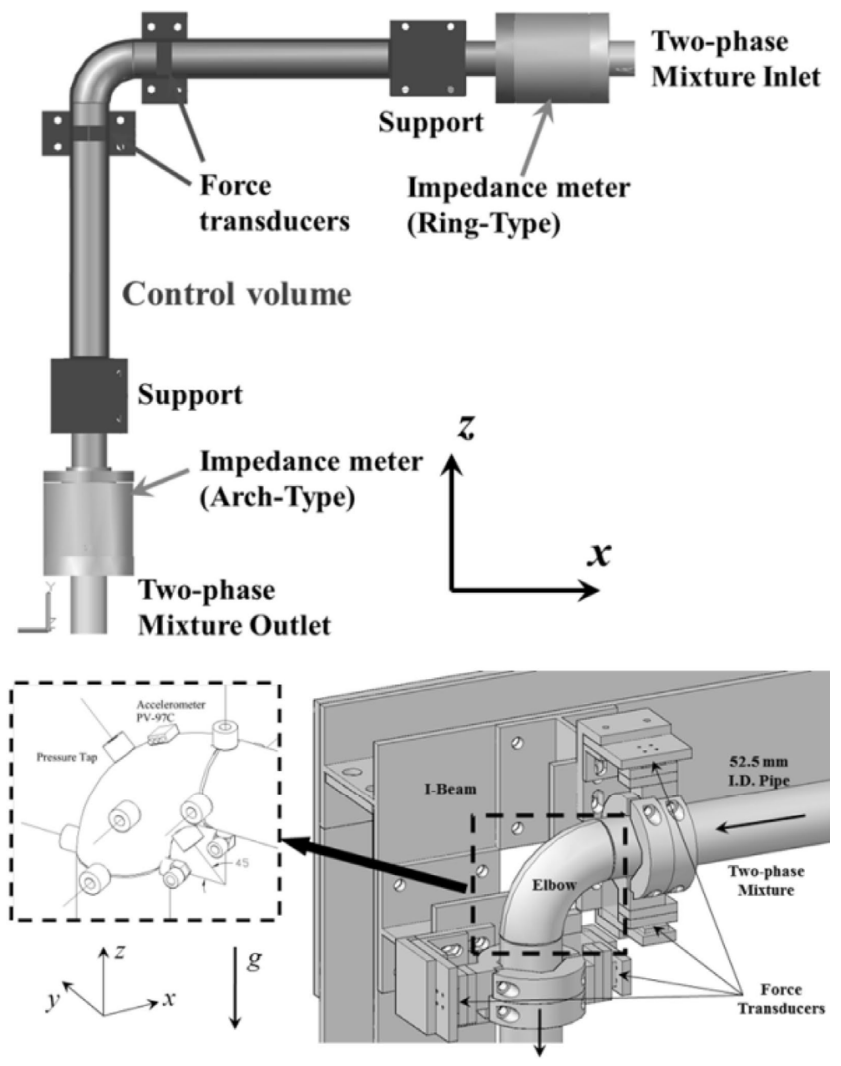

Fig. 5 Two-phase FIV experimental loop (Miwa et al., 2016; reproduced with permission (C) ASME 2016). 
(a)

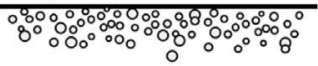

(b)

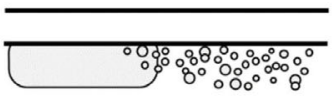

(c)

(d)

(e)

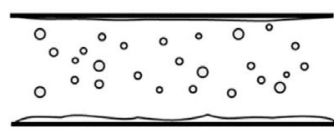

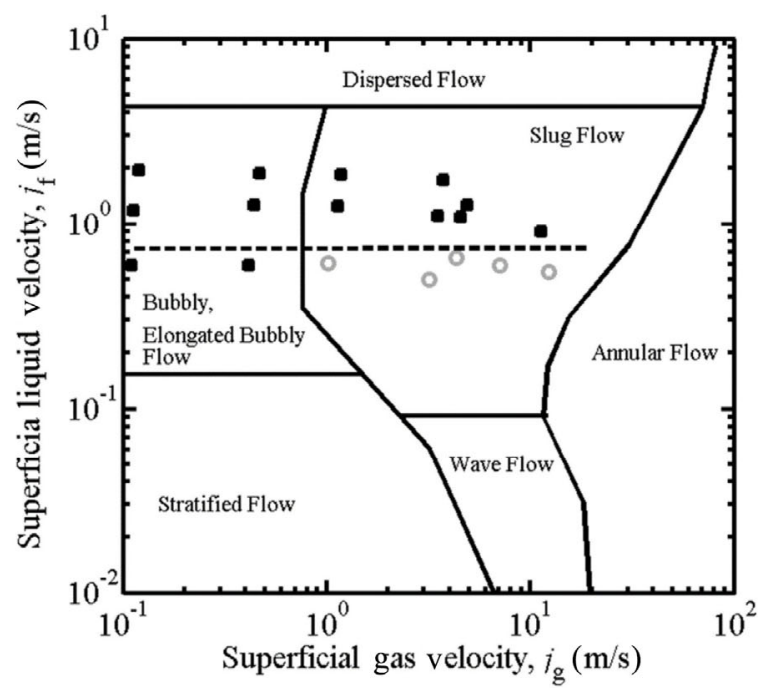

Fig. 6 Experimental conditions for horizontal two-phase FIV (Miwa et al., 2016; reproduced with permission @ ASME 2016).

can be included for the analysis of downwardly acting force fluctuation.

$$
\begin{aligned}
F_{\mathrm{FSx}} \approx & -A \int_{L} \frac{\partial}{\partial t}\left(\tilde{\alpha}_{\mathrm{f}}^{\mathrm{A}} \rho_{\mathrm{f}} j_{\mathrm{t}} \sin \theta\right) \mathrm{d} l \\
& -A j_{\mathrm{t}}^{2}\left(\tilde{\alpha}_{\mathrm{f}}^{\mathrm{A}} \rho_{\mathrm{f}}\right)_{\text {out }}-p_{\text {out }} A+F_{\mathrm{IFx}}
\end{aligned}
$$

In the equation above, $l$ is the axial coordinate of the pipe centerline; $L$ covers the total length of the elbow section. For the upward-horizontal flow turning element, elbow angle $\theta$ is $0^{\circ}$ and $90^{\circ}$ for the upstream and downstream straight pipe sections, respectively. The final term appearing in the right-hand side, $F_{\mathrm{IFx}}$, is the impact force term which must be added for the flow regime with large relative velocity. It can be considered as an additional force term that cannot be explained by the momentum and pressure fluctuations alone, such as impact/collisional force and vortex shedding effects. The way to derive $F_{\mathrm{IFx}}$ will be explained later in this section. For the detailed derivation of impact force term due to upward slug flow regime, reader can refer to Miwa et al. (2015). By applying Fourier transform on the linear force formulation, one obtains force fluctuation spectrum in a following form:

$$
\begin{aligned}
F_{\mathrm{FSx}}(f)= & -A \rho_{\mathrm{f}} j_{\mathrm{t}}^{2} \boldsymbol{A}_{\mathrm{f}}\left(x_{0}, f\right) \mathrm{e}^{-\mathrm{i} 2 \pi \frac{f}{j_{\mathrm{t}}}\left(L_{2}-x_{0}\right)}\left(\frac{\mathrm{i} 2 \pi R f j_{\mathrm{t}} \mathrm{e}^{\mathrm{i} \frac{f^{2} R}{j_{\mathrm{t}}}}+j_{\mathrm{t}}^{2}}{-4 \pi^{2} R^{2} f^{2}+j_{\mathrm{t}}^{2}}\right) \\
& -\boldsymbol{P}_{\text {out }}(f) A
\end{aligned}
$$

Here $\boldsymbol{A}_{\mathrm{f}}, \boldsymbol{F}_{\alpha}, F$, and $\boldsymbol{P}$ are the Fourier transform of liquid fraction $\tilde{\alpha}_{\mathrm{f}}^{\mathrm{A}}$, wave propagation function $f_{\alpha}$, force $F$, and pressure $p$, respectively. By considering the effect of gravity, similar equation can be also derived for the force in $z$ direction.
The above model is capable of predicting fluctuating force frequency and amplitude applied onto elbow structure for flow regimes with homogeneous equilibrium assumption (i.e., negligible relative velocity). However, for the slug flow regime, collisional of the liquid slug against elbow structure generates significant amount of impulsive force and such effect is not considered in the model above. Since slug flow is the most critical flow regime for industrial FIV, proper impact force model must be introduced.

Development of the impact force term for slug flow regime was firstly derived by Miwa et al. (2014a, 2014b) for both upward and horizontal slug. By considering the impact force induced by the collision of liquid plug (or slug) against a wall boundary, a model can be developed for the liquid slug of length $L_{\mathrm{f}}$ being pushed by the external pressure $P_{0}$ in positive $x$-direction. By solving the equation of motion, liquid slug velocity $\left(u_{\mathrm{f}}\right)$ during the collision is expressed as follows.

$$
u_{\mathrm{f}}=\sqrt{\frac{2 P_{0} L_{\mathrm{g}}}{\rho_{\mathrm{f}} L_{\mathrm{f}}}}
$$

Here, $L_{\mathrm{g}}$ is the length of Taylor bubble. Let us now consider the pressure increase during the impact of liquid slug. As is evident from the force generated by the water-hammer effect, impact of liquid slug against the wall boundary creates a sharp pulse, or shock, which travels through the liquid slug at the speed of sound, $c$. Then, change in linear momentum due to the collision can be expressed by the product of mass and change in velocity of the liquid slug. Then, the force exerted on the structure-boundary due to the collision of liquid slug is

$$
F=\rho_{\mathrm{f}} c u_{\mathrm{f}} A
$$


By dividing above expression with cross sectional area, it becomes well-known water-hammer pressure. By replacing liquid-based physical parameters to two-phase flow parameters, above expression can be redefined as follows; let us now substitute the liquid slug velocity defined in Eq. (15) into Eq. (16) and replace the speed of sound, $c$, with two-phase sound velocity, $a_{2 \varphi}$. Then, the impact force due to liquid slug in slug flow regime is expressed as

$$
F_{\mathrm{IF}}=\rho_{2 \varphi} a_{2 \varphi} A \sqrt{\frac{2 P_{0}}{\rho_{2 \varphi}} \frac{L_{\mathrm{g}}}{L_{\mathrm{f}}}}
$$

Here, $a_{2 \varphi}$ is the acoustic sound speed for two-phase flow mixture, replacing the speed of sound term $c$.

In order to obtain slug length, $L_{g}$, one needs to obtain its connection with Sauter mean diameter of the Taylor bubble, which is commonly expressed in terms of interfacial area concentration and void fraction. Here, Sauter mean diameter is defined as

$$
D_{\mathrm{Sm}}=\frac{6 \alpha}{a_{\mathrm{i}}}
$$

In two-phase flow, depending on the behavior of interfacial drag force, bubbles can be classified into two groups, namely, Group-1 and Group-2. Group-1 consists of small scale spherical bubbles, while Group-2 represents relatively large bubbles including distorted bubbles, cap bubbles, and Taylor bubbles (Ishii and Hibiki, 2010). By utilizing the areaaveraging definition,

$$
\langle\alpha\rangle=\frac{1}{A} \int_{A} \alpha \mathrm{d} A
$$

Group-1 and Group-2 area-averaged void fractions should add up to total void fraction, such that following relationship should hold.

$$
\langle\alpha\rangle=\left\langle\alpha_{1}\right\rangle+\left\langle\alpha_{2}\right\rangle
$$

Substituting above expression to the Sauter mean diameter relation, Group-2 Sauter mean diameter can be expressed as follows:

$$
D_{\mathrm{Sm}, 2}=\frac{6\left\langle\alpha_{2}\right\rangle}{\left\langle a_{\mathrm{i} 2}\right\rangle}=\frac{6}{\left\langle a_{\mathrm{i} 2}\right\rangle}\left(\langle\alpha\rangle-\left\langle\alpha_{1}\right\rangle\right)
$$

The interfacial area concentration relation for Group-2 bubble is proposed by Ishii and Mishima (1980) as follows:

$$
\left\langle a_{\mathrm{i} 2}\right\rangle=\frac{4.5 C_{\mathrm{ct}}}{D_{\mathrm{H}}} \frac{\langle\alpha\rangle-\left\langle\alpha_{\mathrm{gs}}\right\rangle}{1-\left\langle\alpha_{\mathrm{gs}}\right\rangle}
$$

$C_{\mathrm{ct}}$ is set to 1.0 for slug flow regime, and this parameter describes roughness that takes into account the effect of irregular gas-liquid interface. For the area-averaged Group-1 interfacial area concentration, following expression can be utilized.

$$
\left\langle a_{\mathrm{i} 1}\right\rangle=\frac{6\left\langle\alpha_{\mathrm{gs}}\right\rangle}{D_{\mathrm{Sm}, 1}} \frac{1-\langle\alpha\rangle}{1-\left\langle\alpha_{\mathrm{gs}}\right\rangle}
$$

Here, $\alpha_{\mathrm{gs}}$ is the Group-1 void fraction within liquid slug. This void fraction value excludes bubbles captured in the liquid film around the Taylor bubble.

$$
\left\langle\alpha_{\mathrm{gs}}\right\rangle=1-\frac{1-\langle\alpha\rangle}{1-\langle\alpha\rangle+\left\langle\alpha_{1}\right\rangle}
$$

By utilizing the correlation proposed by Ozar et al. (2012), the area averaged void fraction for Group- 1 bubble $\left\langle\alpha_{1}\right\rangle$ can be obtained as following:

$$
\left\langle\alpha_{1}\right\rangle= \begin{cases}\langle\alpha\rangle, & \langle\alpha\rangle \leq\left\langle\alpha_{1, \text { max }}\right\rangle \\ \left\langle\alpha_{1, \max }\right\rangle+\left(\frac{\left\langle\alpha_{1, \text { max }}\right\rangle-\left\langle\alpha_{1, \text { base }}\right\rangle}{\left\langle\alpha_{1, \max }\right\rangle-\left\langle\alpha_{\text {crit }}\right\rangle}\right) & \left\langle\alpha_{1, \text { max }}\right\rangle \leq\langle\alpha\rangle \leq\left\langle\alpha_{\text {crit }}\right\rangle \\ \times\left(\langle\alpha\rangle-\left\langle\alpha_{1, \text { max }}\right\rangle\right), & \left\langle\alpha_{\text {crit }}\right\rangle \leq\langle\alpha\rangle \\ \left\langle\alpha_{1, \text { base }}\right\rangle, & \end{cases}
$$

As can be seen, Group-1 void fraction behaves differently depending on the boundary defined with maximum, critical, and base values. The maximum Group-1 void fraction is observed at the transition between bubbly and slug flow regime. Here, spherical and distorted bubbles (Group-1) begin to coalesce and form Taylor bubbles (Group-2). Some Group-1 bubbles are still remained in liquid slug region, and it is represented as $\left\langle\alpha_{1, \text { base }}\right\rangle$. These parameters are dependent on liquid superficial velocity, which is non-dimensionalized by the fluid physical property of liquid density, density difference, and surface tension.

$$
\begin{gathered}
\left\langle\alpha_{1, \max }\right\rangle=\left\{\begin{array}{l}
0.235+0.011\left\langle j_{\mathrm{f}}^{*}\right\rangle, \text { for }\left\langle j_{\mathrm{f}}^{*}\right\rangle \leq 6.1 \\
0.325-0.004\left\langle j_{\mathrm{f}}^{*}\right\rangle, \text { for }\left\langle j_{\mathrm{f}}^{*}\right\rangle \geq 6.1
\end{array}\right. \\
\left\langle\alpha_{\text {crit }}\right\rangle=\left\{\begin{array}{l}
0.511+0.006\left\langle j_{\mathrm{f}}^{*}\right\rangle, \text { for }\left\langle j_{\mathrm{f}}^{*}\right\rangle \leq 6.1 \\
0.645-0.015\left\langle j_{\mathrm{f}}^{*}\right\rangle, \text { for }\left\langle j_{\mathrm{f}}^{*}\right\rangle \geq 6.1
\end{array}\right. \\
\left\langle\alpha_{1, \text { base }}\right\rangle=\left\{\begin{array}{l}
0.099-0.009\left\langle j_{\mathrm{f}}^{*}\right\rangle, \text { for }\left\langle j_{\mathrm{f}}^{*}\right\rangle \leq 6.1 \\
0.05, \quad \text { for }\left\langle j_{\mathrm{f}}^{*}\right\rangle \geq 6.1
\end{array}\right. \\
\left\langle j_{\mathrm{f}}^{*}\right\rangle=\frac{\left\langle j_{\mathrm{f}}\right\rangle}{\left(\frac{\sigma g \Delta \rho}{\rho_{\mathrm{f}}^{2}}\right)^{1 / 4}}
\end{gathered}
$$

It can be assumed that typical slug flow can be explained as the repetition of the slug unit that include Taylor bubbles (Group-2) and spherical bubbles (Group-1). The slug unit is surrounded by the liquid film attached to the pipe diameter $D$. Then, the maximum width of the Taylor bubble can be 
expressed as $\xi D$. Similarly, the liquid film thickness is $D(1-\xi) / 2$. If one treats lengths of Group-1 and 2 bubbles in the slug unit as $L_{1}$ and $L_{2}$, then Group-2 void fraction can be calculated by taking volume averaging of gas over the two-phase mixture.

$$
\alpha_{2}=\frac{\frac{\pi}{4}(\xi D)^{2} L_{2}}{\frac{\pi}{4} D^{2}\left(L_{1}+L_{2}\right)}
$$

In a similar manner, interfacial area concentration of Group-2, which is calculated by dividing total available bubble surface area over the mixture volume, can be expressed as follows:

$$
a_{\mathrm{i} 2}=\frac{2\left[\frac{\pi}{4}(\xi D)^{2}\right]+\pi \xi D L_{2}}{\frac{\pi}{4} D^{2}\left(L_{1}+L_{2}\right)}
$$

By arranging above two relations with Sauter mean formulation, length of the liquid slug can be related with void fraction values, $\alpha_{\mathrm{gs}}, \alpha_{\text {mean }}$, and $\alpha_{2}$.

$$
L_{\mathrm{f}}=\frac{L_{\mathrm{g}}\left(\alpha_{2}-\alpha_{\text {mean }}\right)}{\alpha_{\text {mean }}-\alpha_{\mathrm{gs}}}
$$

Consequently, length scale needed to calculate the impact force term becomes a function of void fraction. Mean void fraction can be determined from regime-dependent correlation of drift flux model, or from the experimental measurement.

In order to determine appropriate value for the ratio between Taylor bubble and channel diameter, parameter $\xi$ can be approximated in several ways. First approach is to utilize the minimum film thickness $\left(\delta_{\text {min }}\right)$ of slug unit structure and void fraction at minimum film thickness $\left(\alpha_{\mathrm{sb}}\right)$ from Mishima-Ishii's model (1984).

$$
\alpha_{\mathrm{sb}}=\frac{D-2 \delta_{\min }}{D}
$$

From Mishima and Ishii, $\alpha_{\mathrm{sb}}$ is defined as

$$
\alpha_{\mathrm{sb}}=\frac{j+\left(1-\alpha_{\mathrm{sb}}\right)^{3 /(2-m)} 3 C_{\mathrm{f}}\left(D / v_{\mathrm{f}}\right)^{-m}\left(\rho_{\mathrm{f}} / \Delta \rho g D\right)^{1 /(m-2)}}{C_{0} j+0.35 \sqrt{\Delta \rho g D / \rho_{\mathrm{f}}}}
$$

$C_{0}$ is the distribution parameter, which can be determined from the drift-flux correlations depending on the shape of flow channel. Another approach would be to assume $10 \%-20 \%$ film thickness surrounding the Taylor bubble and estimate $\xi$ value.

For the horizontal slug, length of the Taylor bubble can be estimated from the time period of passage time $\left(T_{\mathrm{sb}}\right)$ as can be seen from Fig. 7 .

$$
\begin{gathered}
L_{\mathrm{g}}=u_{\mathrm{sb}} T_{\mathrm{sb}}=C_{2} j_{\mathrm{total}} T_{\mathrm{sb}} \\
L_{\mathrm{f}}=\frac{L_{\mathrm{g}}\left(\alpha_{2}-\alpha_{\text {mean }}\right)}{\alpha_{\text {mean }}-\alpha_{1}}
\end{gathered}
$$

The value $C_{2}$ depends on Reynolds number, but for typical slug flow regime, it can be approximated as 1.35.

The two-phase sound speed $\left(a_{2 \varphi}\right)$ can be calculated from homogeneous-equilibrium model. Treating liquid slug as a homogeneous media is reasonable assumption, considering its geometrical characteristics. Then, two-phase sound velocity can be approximated with the form shown below using heat capacity ratio $(\kappa)$, external pressure $\left(P_{0}\right)$, liquid density $\left(\rho_{\mathrm{f}}\right)$, and void fraction $(\alpha)$.

$$
a_{2 \varphi}=\sqrt{\frac{\kappa P_{0}}{\alpha(1-\alpha) \rho_{\mathrm{f}}}}
$$

Final formulation of the impact force term can be expressed as follows:

$$
F_{\mathrm{IF}}(t)=\frac{1}{\sqrt{2}} \rho_{2 \varphi}(t) A_{\text {eff }} \sqrt{\frac{\kappa P_{0}}{\alpha(t)(1-\alpha(t)) \rho_{\mathrm{f}}}} \sqrt{\frac{2 P_{0}}{\rho_{2 \varphi}(t)} \frac{L_{\mathrm{g}}}{L_{\mathrm{f}}}}
$$

By multiplying the factor square root 2, impact force applied onto the 90 degree elbow section can be decomposed to $x$ and $z$-coordinates. The final formulation of the force spectrum predictive model can be expressed as follows:

$$
\begin{aligned}
F_{\mathrm{FSx}}(f)= & -A \rho_{\mathrm{f}} j_{\mathrm{t}}^{2} \boldsymbol{A}_{\mathrm{f}}\left(x_{0}, f\right) \mathrm{e}^{-\mathrm{i} 2 \pi \frac{f}{j_{\mathrm{t}}}\left(L_{2}-x_{0}\right)}\left(\frac{\mathrm{i} 2 \pi R f j_{\mathrm{t}} \mathrm{e}^{\mathrm{i} \frac{f \pi^{2} R}{j_{\mathrm{t}}}}+j_{\mathrm{t}}^{2}}{-4 \pi^{2} R^{2} f^{2}+j_{\mathrm{t}}^{2}}\right) \\
& -\boldsymbol{P}_{\text {out }}(f) A+\int_{-\infty}^{\infty}\left[\frac{1}{\sqrt{2}} \rho_{2 \varphi}(t) A_{\text {eff }} \sqrt{\frac{\kappa P_{0}}{\alpha(t)(1-\alpha(t)) \rho_{\mathrm{f}}}}\right. \\
& \left.\sqrt{\frac{2 P_{0}}{\rho_{2 \varphi}(t)} \frac{L_{\mathrm{g}}}{L_{\mathrm{f}}}}\right] \mathrm{e}^{-\mathrm{i} 2 \pi f t} \mathrm{~d} t
\end{aligned}
$$

The formulation above is applicable for bubbly to churn flow regime in small diameter pipe (less than $10 \mathrm{~cm}$ inner diameter for atmospheric pressure). When the area-averaged void fraction value exceeds 0.3 , Group-2 bubble is formed and impact force term shown in the very last of right-hand side is automatically included into the formulation.

Above formulation can be applied for different from regime in horizontal two-phase flow, such as stratifiedwavy flow regime, as was reported by Miwa et al. (2016). As can be seen from the schematic (Fig. 8), stratified-wavy flow possesses large amplitude wave crest, which acts as a source of collision force when interacted with pipe turning element. This flow regime generates high amplitude vibration, comparable to slug flow regime (Fig. 9). Considering a simple geometry shown in Fig. 10, the impact force named 

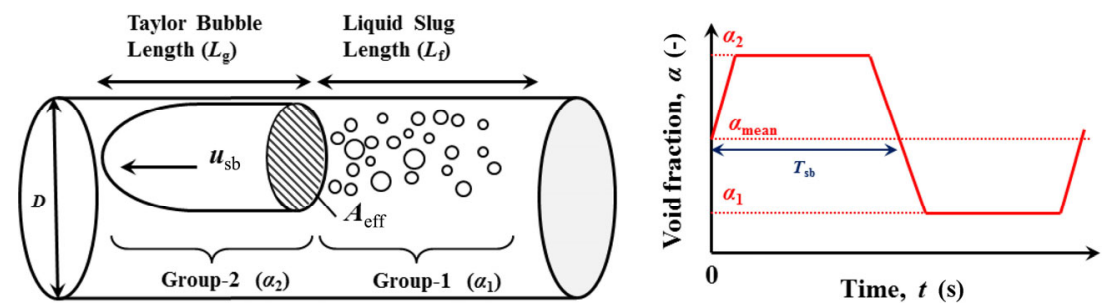

Fig. 7 Schematic of the horizontal slug unit, consist of Taylor bubble (Group-2) and distorted bubble (Group-1) (Miwa et al., 2014a; reproduced with permission (c) The Japan Society of Mechanical Engineers 2014).

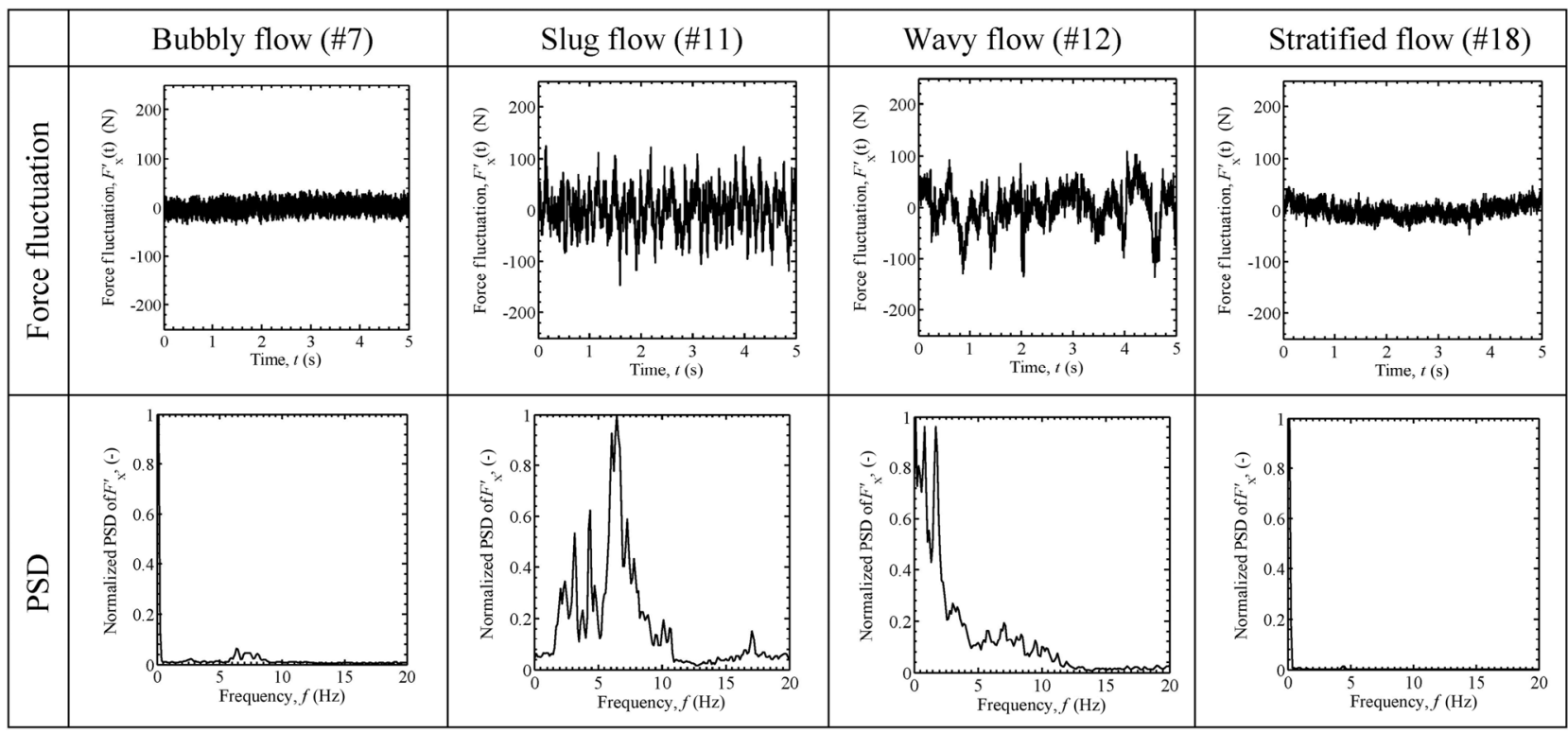

Fig. 8 Force fluctuation measurement results for flow regimes including bubbly flow (\#7), slug flow (\#11), wavy flow (\#12), and stratified flow (\#18) (Miwa et al., 2014a; reproduced with permission () The Japan Society of Mechanical Engineers 2014).
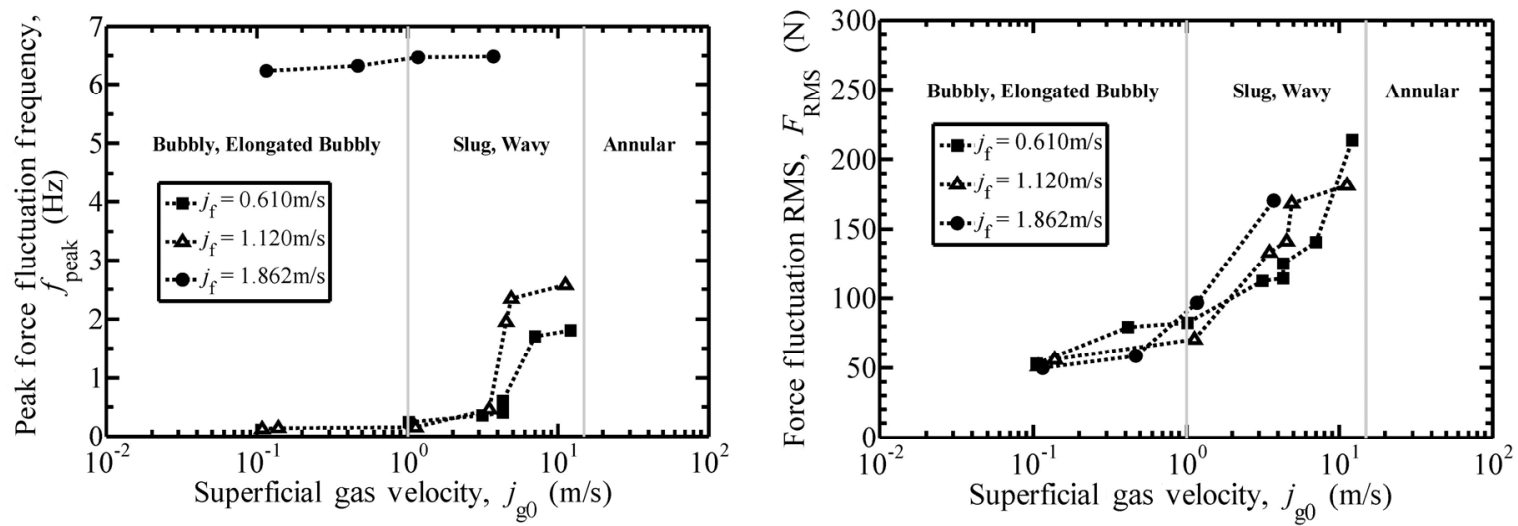

Fig. 9 Change in force spectrum peak frequency (top) and peak PSD amplitude (bottom) with respect to inlet superficial gas velocity (Miwa et al., 2014b; reproduced with permission (C) The Japan Society of Mechanical Engineers 2014).

as wave collisional force was successfully derived.

$$
\begin{aligned}
F_{\mathrm{WCF}}= & \rho_{\mathrm{f}} c_{\text {sound }}\left\{\left[\frac{-\left(v_{\mathrm{g}}-v_{\mathrm{f}}\right)^{2}}{\alpha / \rho_{\mathrm{g}}+(1-\alpha) / \rho_{\mathrm{f}}}+\left(\rho_{\mathrm{f}}-\rho_{\mathrm{g}}\right) g D\right]^{1 / 2}\right. \\
& \left.\cdot\left(\frac{\rho_{\mathrm{g}}}{\alpha}+\frac{\rho_{\mathrm{f}}}{1-\alpha}\right)^{-1 / 2}+V_{0}\right\}\left[\frac{\pi D^{2}}{4}\left(\alpha_{\max }-\alpha\right)\right]
\end{aligned}
$$

\section{Conclusions and future perspectives}

As was reviewed in the article, FIV in plant component remains to be a challenging issue, particularly in energy sector such as nuclear and petroleum industries. As the projected global energy demand continue rising, existing 


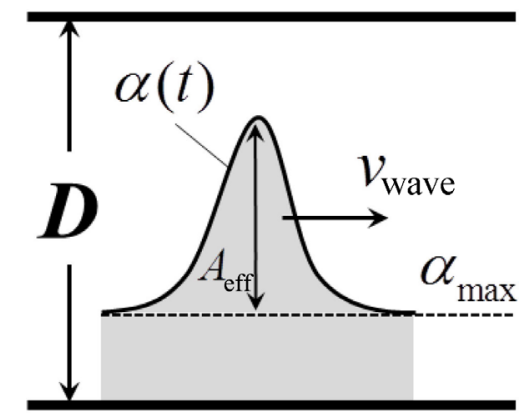

Fig. 10 Simplified model for the impact force generated by the wave crest (Miwa et al., 2016; reproduced with permission (c) ASME 2016).

power plants will likely be operated at maximum capacity in developed countries. Hence, investigation of FIVs in plant component will continue to be a vital topic for thermalhydraulic and structural engineers. Due to the advancement of multiphase flow models embedded in commercial CFDs, more accurate simulation of multiphase flow field is now possible. Key challenges will be to efficiently link these tools to the structural analysis package for the multi-dimensional FIV analysis. Until such tools become reliable, experimental database development at laboratory scale will continue to be significant. Especially with the advancement of multiphase flow measurement technique such as local probe (Pettigrew et al., 2014), interfacial area concentration measurement (Ishii and Hibiki, 2010), and non-intrusive vibration measurement tools, more detailed and local database can be generated.

To handle these databases efficiently, utilization of machine learning approach is also promising approach (Saito et al., 2018). As were pointed out by various researchers, FIV due to multiphase flow is a flow regime specific problem. For the two-phase flow FIV analysis, researchers are still relying on the flow regime map created back in the 1970s and 1980s, which can be subjective. Adaptation of such approach for FIV analysis will be beneficial to understand the nature of FIV generated by multiphase flow at the plant scale.

\section{References}

Al-Khalifa, H. A., Oshinowo, L., Al-Saif, O. A. 2016. Transient multiphase simulation in separator vessel internals design in Saudi Aramco. In: Proceedings of the ASME 2016 International Mechanical Engineering Congress and Exposition, IMECE2016-65250.

Álvarez-Briceño, R., Kanizawa, F. T., Ribatski, G., de Oliveira, L. P. R. 2018. Validation of turbulence induced vibration design guidelines in a normal triangular tube bundle during two-phase crossflow. J Fluid Struct, 76: 301-318.

American Society of Mechanical Engineers (ASME). 1998. ASME boiler and pressure vessel code Section III, Nonmandatory Appendix N, Subarticle N-1300, Flow-Induced Vibration of Tubes and Tube Banks 1998 with 2000 Addendum.
Belfroid, S. P. C., Nennie, E., Lewis, M. 2016. Multiphase forces on bends-Large scale 6-inch experiments. In: Proceedings of the SPE Annual Technical Conference and Exhibition, SPE-181604-MS.

Blevins, R. D. 1990. Flow-Induced Vibration, 2nd edn. New York: Van Nostrand Reinhold.

Blevins, R. D. 2018. Nonproprietary flow-induced vibration analysis of San Onofre nuclear generating station replacement steam generators to ASME code section III appendix N.J Pressure Vessel Technol, 140: 034502.

Cabrera-Miranda, J. M., Paik, J. K. 2019. Two-phase flow induced vibrations in a marine riser conveying a fluid with rectangular pulse train mass. Ocean Eng, 174: 71-83.

Da Silva, B. L., dos Santos, C. M., Bianchi, P., Henry, N., Meier, F., Oliveira, E., Martiganoni, W. P. 2016. Flow simulations solve WHRSG vibration issues. Oil Gas J, 114: 72.

Diwakar, P., Prakash, A., Thomas, C. 2017. Flow induced vibration of equipment internals in a two-phase gas/liquid flow. In: Proceedings of the ASME 2017 Pressure Vessels and Piping Conference, PVP2017-65812.

Giraudeau, M., Mureithi, N. W., Pettigrew, M. J. 2013. Two-phase flow-induced forces on piping in vertical upward flow: Excitation mechanisms and correlation models. J Pressure Vessel Technol, 135: 030907.

Ishii, M., Hibiki, T. 2010. Thermo-Fluid Dynamics of Two-Phase Flow. Springer Science \& Business Media.

Ishii, M., Mishima, K. 1980. Study of two-fluid model and interfacial area. NUREG/CR-1873; ANL-80-111. Argonne National Lab., IL, USA.

Japan Society of Mechanical Engineers (JSME). 2018. Flow Induced Vibrations-Classification and Lessons from Practical Experiences, 3rd edn. Tokyo: Gihodo Publishing Co.

Li, F., Cao, J., Duan, M., An, C., Su, J. 2016. Two-phase flow induced vibration of subsea span pipeline. In: Proceedings of the 26th International Ocean and Polar Engineering Conference, ISOPE-I-16-333.

Liu, Y., Miwa, S., Hibiki, T., Ishii, M., Morita, H., Kondoh, Y., Tanimoto, K. 2012. Experimental study of internal two-phase flow induced fluctuating force on a 90 elbow. Chem Eng Sci, 76: 173-187.

Mishima, K., Ishii, M. 1984. Flow regime transition criteria for upward two-phase flow in vertical tubes. Int J Heat Mass Tran, 27: 723-737.

Miwa, S., Hibiki, T., Mori, M. 2016. Analysis of flow-induced vibration due to stratified wavy two-phase flow. J Fluid Eng, 138: 091302.

Miwa, S., Liu, Y., Hibiki, T., Ishii, M., Kondo, Y., Morita, H., Tanimoto, K. 2014a. Study of unsteady gas-liquid two-phase flow induced force fluctuation (Part 2: Horizontal-downward two-phase flow). Trans JSME, 80: TEP0046. (in Japanese)

Miwa, S., Liu, Y., Hibiki, T., Ishii, M., Kondo, Y., Morita, H., Tanimoto, K. 2014b. Study of unsteady gas-liquid two-phase flow induced force fluctuation (Part 1: Evaluation and modeling of two-phase flow induced force fluctuation). Trans JSME, 80: FE0005. (in Japanese)

Miwa, S., Mori, M., Hibiki, T. 2015. Two-phase flow induced vibration in piping systems. Prog Nucl Energ, 78: 270-284.

Miwa, S., Yamamoto, Y., Chiba, G. 2018. Research activities on nuclear reactor physics and thermal-hydraulics in Japan after 
Fukushima-Daiichi accident. J Nucl Sci Technol, 55: 575-598.

Nakamura, T., Shiraishi, T., Ishitani, Y., Watakabe, H., Sago, H., Fujii, T., Konomura, M. 2005. Flow-induced vibration of a large-diameter elbow piping based on random force measurement caused by conveying fluid (visualization test results). In: Proceedings of the ASME 2005 Pressure Vessel and Piping, PVP2005-71277.

Olala, S., Mureithi, N. W., Sawadogo, T., Pettigrew, M. J. 2014. Streamwise fluidelastic forces in tube arrays subjected to two-phase flows. In: Proceedings of the ASME 2014 Pressure Vessels and Piping Conference, PVP2014-28153.

Ong, Z. C., Eng, H. C., Noroozi, S. 2017. Non-destructive testing and assessment of a piping system with excessive vibration and recurrence crack issue: An industrial case study. Eng Fail Anal, 82: 280-297.

Ortiz-Vidal, L. E., Mureithi, N. W., Rodriguez, O. M. H. 2017. Vibration response of a pipe subjected to two-phase flow: Analytical formulations and experiments. Nucl Eng Des, 313: 214-224.

Ozar, B., Dixit, A., Chen, S. W., Hibiki, T., Ishii, M. 2012. Interfacial area concentration in gas-liquid bubbly to churn-turbulent flow regime. Int J Heat Fluid Fl, 38: 168-179.

Paidoussis, M. P. 1970. Dynamics of tubular cantilevers conveying fluid. J Mech Eng Sci, 12: 85-103.

Pettigrew, M. J., Besner, B., Mureithi, N. W., Lafrance, T., Patrick, J. M. 2014. Development of fiber-optic probes to measure two-phase flow dynamic parameters in support of flow-induced vibration studies. In: Proceedings of the ASME 2014 Pressure Vessels and Piping Conference, PVP2014-28106.

Saito, Y., Torisaki, S., Miwa, S. 2018. Two-phase flow regime identification using fluctuating force signals under machine learning techniques. In: Proceedings of the 2018 26th International Conference on Nuclear Engineering, ICONE26-81288.

Schulkes, R. 2011. Slug frequency revisited. In: Proceedings of the 15th International Conference on Multiphase Production Technology, BHR-2011-H1
Setyawan, A., Indarto, Deendarlianto. 2016. The effect of the fluid properties on the wave velocity and wave frequency of gas-liquid annular two-phase flow in a horizontal pipe. Exp Therm Fluid Sci, 71:25-41.

Wang, L., Yang, Y. R., Li, Y. X., Wang, Y. T. 2018a. Dynamic behaviours of horizontal gas-liquid pipes subjected to hydrodynamic slug flow: Modelling and experiments. Int J Pres Ves Pip, 161: 50-57.

Wang, L., Yang, Y. R., Li, Y. X., Wang, Y. T. 2018b. Resonance analyses of a pipeline-riser system conveying gas-liquid two-phase flow with flow-pattern evolution. Int J Pres Ves Pip, 161: 22-32.

Wang, L., Yang, Y. R., Liu, C., Li, Y. X., Hu, Q. H. 2018c. Numerical investigation of dynamic response of a pipeline-riser system caused by severe slugging flow. Int J Pres Ves Pip, 159: 15-27.

Xu, X. P., Liu, M. Y., Ma, Y., An, M. 2016. Effects of fluidized solid particles on vibration behaviors of a graphite tube evaporator with an internal vapor-liquid flow. Appl Therm Eng, 100: 1229-1244.

Open Access This article is licensed under a Creative Commons Attribution 4.0 International License, which permits use, sharing, adaptation, distribution and reproduction in any medium or format, as long as you give appropriate credit to the original author(s) and the source, provide a link to the Creative Commons licence, and indicate if changes were made.

The images or other third party material in this article are included in the article's Creative Commons licence, unless indicated otherwise in a credit line to the material. If material is not included in the article's Creative Commons licence and your intended use is not permitted by statutory regulation or exceeds the permitted use, you will need to obtain permission directly from the copyright holder.

To view a copy of this licence, visit http://creativecommons.org/licenses/by/4.0/. 